\title{
Multi-band Observation of TeV Supernova Remnants
}

\author{
W. W. $\operatorname{Tian}^{1}$, D. A. Leahy ${ }^{2}$ and Hongquan $\mathrm{Su}^{1}$ \\ ${ }^{1}$ National Astronomical Observatories, CAS, Beijing 100012, China email: tww@bao.ac.cn \\ ${ }^{2}$ Department of Physics \& Astronomy, University of Calgary, Alberta T2N 1N4, Canada
}

\begin{abstract}
We study several TeV Supernova Remnants (SNRs W51C, CTB 37A, CTB 37B and G353.6-0.7) by radio and X-ray observations. We utilize neutral hydrogen (HI) $21 \mathrm{~cm}$ line data to measure their kinematic distances, and use the CO line survey sensitive to molecular hydrogen clouds to validate these distance measurements and understand their relation to the TeV SNRs. Our study show that the TeV $\gamma$-ray emission from W51C should not be associated with the high-velocity HI clouds; CTB 37A and CTB 37B are at different distances and are only by chance nearby each other on the sky; the extended TeV emission from G353.6-0.7 possibly originates from the interaction between the SNR shock and the adjacent CO clouds.
\end{abstract}

Keywords. ISM:supernova remnants - ISM:HII regions - ISM:lines and bands - cosmic rays

\section{Background}

Galactic cosmic Rays(CRs) have been believed to originate from supernova remnants (SNRs). Recent very-high-energy observations show that more than 10 Galactic Supernova Remnants (SNRs) emit TeV $\gamma$-rays (http://tevcat.uchicago.edu/). However, it is still unclear whether the charged particles emitting the $\mathrm{TeV} \gamma$-rays are accelerated protons or electrons. Since 2007, we have studied TeV SNRs (e.g. W51C, CTB 37A/B and G353.6-0.7) by multi-band observation which has proven to be robust in shedding light on $\gamma$-ray sources' nature. We have utilized recently-released neutral hydrogen (HI) $21 \mathrm{~cm}$ line data from international Galactic plane survey (Stil et al.2006) and the ${ }^{13} \mathrm{CO}$ line data from the Galactic ring survey (Jackson et al. 2006) to understand their relation to the TeV SNRs.

\section{Results}

\subsection{W51C/HESS J1923+141}

W51C is located in the strong radio source complex W51. W51 contains components of thermal emission from HII regions (W51A/B) and non-thermal emission from SNR W51C (Koo et al. 2005). The recently-detected TeV $\gamma$-ray source HESS J1923+141 coincides with Supernova Remnant (SNR) W51C and the star forming region W51B (Aleksic et al. 2012). W51C was suggested to be posssibly interacting with nearby hydrogen molecular/atomic clouds at radial velocity of $\sim 70 \mathrm{~km} \mathrm{~s}^{-1}$ surrounding the SNR's northwestern shell (Koo \& Moon 1997). Two compact $1720 \mathrm{MHz} O \mathrm{OH}$ masers have been detected at W51B and the north-western edge of the W51C shell (Hewitt et al. 2008). The masers are caused by shocks either from the SNR W51C or from the star forming region W51B.

Our observation detects high-velocity (HV) HI clouds $\left(>83 \mathrm{~km} \mathrm{~s}^{-1}\right)$ which coincide with W51B. We find that the clouds are behind W51B and W51C is possibly in front of W51B. This argues against previous claims that the SNR has shocked the HV HI clouds. 
In addition, we think that the observed nearby $\mathrm{OH}$ masers from dense molecular gas are likely associated with the star forming region in W51B but not W51C. These new results show clear absence of evidence of molecular or atomic gas interacting with W51C, thus casting doubt on a hadronic origin for the $\mathrm{GeV} / \mathrm{TeV}$ emission from W51C. We think W51C is similar to Tycho SN 1572 (Tian \& Leahy 2011) which is not interacting with HI clouds. We also suggest a distance of about $4.3 \mathrm{kpc}$ for W51C, smaller than the tangent point distance of $5.5 \mathrm{kpc}$ in that direction, but still in the Sagittarius spiral arm.

\subsection{CTB 37A/HESS J1714-385 and CTB 37B/HESS J1713-381}

SNRs CTB 37A and CTB 37B are associated with the TeV source HESS J1714-385 and HESS J1713-381 respectively. We use the $1420-\mathrm{MHz}$ radio continuum and 21-cm HI data from the Southern Galactic Plane Survey (SGPS, Haverkorn et al. 2006) and build 21-cm HI absorption spectrum to constrain kinematic distances to the SNRs. Our measurements show that CTB 37A has a distance in the range 6.3-9.5 kpc (previously $\sim 11.3 \mathrm{kpc}$ ) and an HI column density of $\sim 7.1 \times 10^{21} \mathrm{~cm}^{2}$. CTB 37B is at a distance of $\sim 13.2 \mathrm{kpc}$ (previously 5-9 kpc) and has an HI column density of $\sim 8.3 \times 10^{21} \mathrm{~cm}^{2}$. CTB $37 \mathrm{~A}$ and CTB 37B are at different distances and are only by chance nearby each other on the sky (Tian \& Leahy 2012).

\subsection{G353.6-0.7/HESS J1731-347}

There is a rare class of TeV-emitting SNRs which have non-thermal X-ray and TeV gamma-ray shells (i.e. SNRs G266.2-1.2, G347.3-0.5, G315.2-2.3 and SN1006). SNR G353.6-0.7/HESS 1731-347 is the newest one of the class (Tian et al. 2008, Abramowski et al. 2011). We study the system by using radio data from the SGPS and Delinha CO observation, X-ray data from XMM-Newton, Suzaku and Chandra observations. We detect the extended hard non-thermal X-ray emission which is coincident with HESS J1731-347 and the shell of the radio remnant, and no thermal X-ray emission from the SNR at a significant level (Bamba et al. 2012). Based on the probable association between the hard $\mathrm{X}$-ray and $\gamma$-ray emissions and likely association between the CO cloud and the SNR, we conclude that the extended $\mathrm{TeV}$ emission likely originates from the interaction between the SNR shock and adjacent CO cloud.

\section{Acknowledgements}

We thank supports from the NSFC(011241001, 211381001), BaiRen program of the CAS(034031001), and China Ministry of Science and Technology's Program (2013CB837901, 2012CB821800). This publication was partly supported by a grant from the John Templeton Foundation and National Astronomical Observatories of the CAS.

\section{References}

Abramowski, A., Acero, F., Aharonian, F., et al. 2011, ApJ, 735, 12

Aleksic, J., Alvarez, E. A., Antonelli, L. A., et al. 2012, A $\& A$, 541, 13

Bamba, A., Pühlhofer, G., Acero, F., et al. 2012, ApJ, 756, 149

Hewitt, J. W., Yusef-Zadeh, F., \& Wardle, M. 2008, ApJ, 683, 189

Haverkorn, M., Gaensler, B. M., McClure-Griffiths, N. M., et al. 2006, ApJS, 167, 230

Jackson, J. M., Rathborne, J. M., Shah, R. H., et al. 2012, ApJ, 163, 145

Koo, B. C., Moon,D. S. 1997, ApJ, 475, 194

Koo, B. C., Kim, K. T., Seward, F. D., \& Lee, J. J. 2005, ApJ, 633, 946

Stil, J. M., Taylor, A. R., Dickey, J. M. et al. 2006, AJ, 132, 1158

Tian, W. W., Leahy, D. A., Haverkorn, M., \& Jiang, B. 2008, ApJ, 679, L85

Tian, W. W. \& Leahy, D. A. 2011, ApJL, 729, 215

Tian, W. W. \& Leahy, D. A. 2012, MNRAS, 421, 2593 